\title{
Metabolites involved in glycolysis and amino acid metabolism are altered in short children born small for gestational age
}

\author{
Philip G. Murray ${ }^{1,2}$, Imogen Butcher ${ }^{1,2}$, Warwick B. Dunn ${ }^{1,3,4}$, Adam Stevens ${ }^{1,2}$, Reena Perchard ${ }^{1,2}$, Daniel Hanson ${ }^{1,2}$, \\ Andrew Whatmore ${ }^{1,2}$, Melissa Westwood ${ }^{1,5}$ and Peter E. Clayton ${ }^{1,2}$
}

BACKGROUND: Later life metabolic dysfunction is a wellrecognized consequence of being born small for gestational age (SGA). This study has applied metabolomics to identify whether there are changes in these pathways in prepubertal short SGA children and aimed to compare the intracellular and extracellular metabolome in fibroblasts derived from healthy children and SGA children with postnatal growth impairment. METHODS: Skin fibroblast cell lines were established from eight SGA children (age 1.8-10.3 y) with failure of catch-up growth and from three healthy control children. Confluent cells were incubated in serum-free media and the spent growth medium (metabolic footprint), and intracellular metabolome (metabolic fingerprint) were analyzed by gas-chromatography mass spectrometry.

RESULTS: Nineteen metabolites were significantly altered between SGA and control cell lines. The greatest fold difference (FD) was seen for alanine (fingerprint FD, SGA: control $0.3, P=0.01$ and footprint FD $=0.19, P=0.01$ ), aspartic acid (fingerprint FD $=5.21, P=0.01$ ), and cystine (footprint FD = $1.66, P=0.02)$. Network analysis of the differentially expressed metabolites predicted inhibition of insulin as well as growth (ERK) signaling in SGA cells.

CONCLUSION: This study indicates that changes in cellular metabolism associated with both growth failure and insulin insensitivity are present in prepubertal short children born SGA.

$\mathbf{T}$ here are approximately 700,000 children born in the United Kingdom each year (1). Using a definition of small for gestational age (SGA) as a birth weight more than 2 SD below mean birth weight, there are approximately 16,000 SGA children born each year. SGA neonates can broadly be divided into those who are constitutionally small (i.e., small in comparison to population standards but normal for familial and ethnic background) and those with pathological growth impairment (2). Intrauterine growth restriction is a term applied to fetuses and neonates with an estimated fetal weight or birth weight/length $<10$ th percentile due to pathological growth restriction due to adverse genetic or environmental influences (3). Around $10 \%$ of children born
SGA will fail to show catch-up growth over the first $2 \mathrm{y}$ of life, leaving approximately 1,600 children in the United Kingdom who remain short (height standard deviation score (SDS) <-2) at $2 \mathrm{y}$ of age (4). As well as growth impairment children born SGA are at increased risk of cardiovascular disease, hypertension, hyperlipidaemia, and type 2 diabetes in adulthood (5).

The causes of children being born SGA are numerous and include maternal, placental, and fetal factors (5). There are a small number of monogenic causes for a child to be born SGA and experience poor postnatal growth (e.g., 3-M syndrome, Bloom syndrome, Microcephalic Osteodysplastic dwarfism type II, IGF-IR mutations, Seckel syndrome, and Mulibrey nanism) (6).

Metabolomics provides a number of advantages over single analyte measurement, for example, high-throughput analytical strategies and the provision of a dynamic and sensitive measure of all metabolites that contribute to the phenotype being examined (7). In mammals, metabolomics is typically applied in the discovery of novel biomarkers of disease (8-11), drug efficacy (12), or toxicity (10), or is used to understand molecular pathophysiological mechanisms (12-14). In mammalian systems, metabolites act as building blocks for larger molecules (e.g., proteins) and structures (e.g., lipids in cell walls) and they act to regulate biochemical processes (e.g., allosterism) and as signaling molecules (7). When studying cultured mammalian cells and tissues, two separate but linked metabolomes can be studied, the intracellular metabolome (metabolic fingerprint) and the extracellular metabolome (metabolic footprint). The metabolic fingerprint allows a snapshot of cellular metabolism to be obtained while the footprint reflects the effects of metabolite uptake and secretion.

This study has used an established ex-vivo skin fibroblast cell model as previous work has shown this to be a good model for examining growth disorders (15-17). The aim of this study was to elucidate potential novel pathophysiological mechanisms associated with (i) failure of postnatal catch-up growth in SGA children and (ii) the well-recognized predisposition to metabolic morbidity and diabetes in later life in short children born SGA (18). A model culture system using ex-vivo skin fibroblast cells was applied in this study as previous work has shown this to be a good model for examining growth disorders (15-17). While

\footnotetext{
IInstitute of Human Development, University of Manchester, Manchester, UK; ${ }^{2}$ Centre for Paediatrics and Child Health, University of Manchester, Manchester, UK; ${ }^{3}$ Manchester Centre for Integrative Systems Biology, University of Manchester, Manchester, UK; ${ }^{4}$ School of Biosciences, University of Birmingham, Birmingham, UK; ${ }^{5}$ Maternal and Fetal Health Research Centre, University of Manchester, Manchester, UK. Correspondence: Philip G. Murray (Philip.Murray@manchester.ac.uk) Received 19 August 2015; accepted 2 February 2016; advance online publication 4 May 2016. doi:10.1038/pr.2016.72
} 
previous studies have examined serum and urine metabolomics in SGA/IUGR children (19-21) by utilizing a cell-based model, it is possible to gain deeper insight into intracellular metabolism, potentially yielding useful information on the mechanisms underlying the growth impairment, and metabolic changes seen in these patients.

\section{RESULTS}

In the metabolic fingerprint study, 93 unique chromatographic peaks (related to 38 unique and identified metabolites) were detected in the samples from both control and SGA cells. In the metabolic footprint study, 95 unique chromatographic peaks were detected in all samples studied; these related to 39 unique and identified metabolites.

Table 1. Metabolites up- or downregulated comparing the metabolic fingerprint and metabolic footprint from SGA fibroblasts to control fibroblasts

\begin{tabular}{lccc}
\hline Metabolite & Ratio & $P$ value & Source \\
\hline Alanine & 0.33 & 0.014 & Fingerprint \\
Threonine & 1.25 & 0.041 & Fingerprint \\
Glutamic acid & 1.39 & 0.025 & Fingerprint \\
Ornithine & 1.44 & 0.014 & Fingerprint \\
Citric acid & 1.53 & 0.041 & Fingerprint \\
Serine & 1.65 & 0.014 & Fingerprint \\
Fructose & 1.8 & 0.014 & Fingerprint \\
Aspartic acid & 5.21 & 0.014 & Fingerprint \\
Alanine & 0.19 & 0.014 & Footprint \\
Cysteine & 0.32 & 0.014 & Footprint \\
2-methyl-3-hydroxybutanoic acid & 0.53 & 0.049 & Footprint \\
3-methylpentanoic acid & 0.54 & 0.014 & Footprint \\
Hexanoic acid & 0.58 & 0.041 & Footprint \\
Phenylalalnine & 0.65 & 0.025 & Footprint \\
Aminomalonic acid & 0.71 & 0.041 & Footprint \\
Trimethylamine-N-oxide & 1.18 & 0.025 & Footprint \\
Phosphate & 1.26 & 0.041 & Footprint \\
Pyruvic acid & 1.42 & 0.025 & Footprint \\
Cystine & 1.66 & 0.025 & Footprint \\
\hline SGA fibrats & & & \\
& & &
\end{tabular}

SGA fibroblasts $(n=8)$ and control fibroblasts $(n=3)$. The fold change is calculated as median-SGA/median-control.

SGA, small for gestational age.
Comparing the metabolome (fingerprint and footprint) of all the SGA patient cells $(n=8)$ to controls $(n=3), 29$ metabolic peaks representing 8 unique and identified metabolites in the fingerprint and 11 unique and identified metabolites in the footprint were statistically significantly up- or downregulated (Table 1). The amino acid alanine was downregulated in the SGA cell lines in both footprint (fold difference $(F D)=0.33$ ) and fingerprint $(\mathrm{FD}=0.19)$. There were five amino acids upregulated in the metabolic fingerprint of the SGA cell lines: threonine, ornithine, serine, aspartic acid, and glutamic acid. The only metabolites not classified as amino acids and identified in the fingerprint were the carbohydrate fructose, which was upregulated in the SGA cell lines $(F D=1.8)$ and the Krebs cycle intermediary, citric acid, which was also upregulated $(\mathrm{FD}=1.53)$. In the metabolic footprint data, the amino acids cysteine $(\mathrm{FD}=0.32)$ and phenylalanine $(\mathrm{FD}=0.65)$ were downregulated in SGA cell lines while cystine was upregulated $(\mathrm{FD}=1.66)$. Pyruvic acid was upregulated and 2-methyl-3-hydroxybutanoic acid and 3-methylpentanoic acid were downregulated in the SGA cell lines.

Subgroup analysis comparing the 3-M syndrome and non3-M syndrome patients both to control and to each other also identified differences in the metabolomics fingerprint and footprint (see Supplementary Tables S1-S3 online). Of the 18 metabolic peaks identified as differentially regulated between control and 3-M patients, only 3 (inositol-1-phosphate, glutamine, and 2-oxopropanoic acid) were not also identified as dysregulated in the whole group analysis. There were also 18 metabolites significantly different between the non-3-M patients and controls of which 3 (myo-inositol, lysine, and phosphate) were not identified as being dysregulated between all patients and controls. There were five metabolic peaks in the fingerprint (alanine, aspartic acid, fructose, ornithine, and serine) and three in the footprint (3-methylpentanoic acid, alanine, and cysteine) significantly different to controls for all the patients as well as in both the subgroup analyses of 3-M and non $3-\mathrm{M}$ patients. There were 21 different metabolites significantly up- or downregulated between the 3-M and non-3-M patients (Supplementary Table S3 online) including 9 metabolites in the footprint and 11 in the fingerprint.

Metabolic Pathway Enrichment Analysis, using MetaboAnalyst (22) was performed by including all unique and statistically significant metabolites detected in the metabolic fingerprint and footprint samples when comparing all the SGA patient cells to controls. Five metabolic pathways showed enrichment with a false discovery rate of $q<0.05$ (see Table 2).

Table 2. Metabolic pathways enrichment analysis using the metabolic fingerprint and metabolic footprint metabolites identified as significantly up-/downregulated between control and small for gestational age cells

\begin{tabular}{lccl} 
Pathway name & $\begin{array}{c}\text { Number of metabolites } \\
\text { statistically significant }\end{array}$ & FDR (q value) & Metabolites \\
\hline Cyanoamino acid metabolism & 3 & 0.0074 & Glycine, serine, alanine \\
Glycine, serine and threonine metabolism & 4 & 0.0093 & Threonione, glycine, serine, pyruvate \\
Alanine, aspartate and glutamate metabolism & 3 & 0.0129 & Glutamine, asparate, pyruvate \\
Methane metabolism & 3 & 0.0291 & Trimethylamine-N-oxide, glycine, serine \\
Nitrogen metabolism & 3 & 0.0362 & Phenylalanine, glutamine, glycine \\
\hline
\end{tabular}


An interaction network formed from the metabolites identified as differentially regulated between all SGA patient and control cell lines contained PI3K, AKT, p38, and ERK in addition to molecules in the proinsulin-insulin pathway (Figure 1). This interaction network predicts inhibition insulin activity. Biological functions associated with the differentially regulated metabolites included carbohydrate metabolism, cell-cell signaling, cell growth and cell cycle (see Figure 2).

The identification of these protein kinases in the network led us to validate their involvement by examining the activation of kinases in response to IGF-I using a phospho-kinase array in the cell lines. Treatment with IGF-I led to a significant change $(P<0.05)$ in phosphorylation of nine proteins in control cells (ERK1, ERK2, JNK1, JNK2, pan JNK, p38, RSK2, pan AKT, and MSK2) and seven proteins in the SGA cells (ERK1, JNK1, p38, RSK1, RSK2, AKT2, and MSK2) (see Figure 3). These data imply that in control but not in SGA cells IGF-I preferentially activates ERK2 and JNK2 while, in SGA but not controls,
IGF-I preferentially activates RSK1. Both ERK2 and JNK are contained within the interaction network formed from the differentially regulated metabolites (Figure 1) and are linked via glutamic acid.

\section{DISCUSSION}

The primary aim of this study was to identify metabolomic changes in fibroblasts from children with a severe SGA/growth impairment syndrome and to identify pathogenic mechanisms linking these metabolomic changes to short stature and/ or early markers of metabolic disease. There are limited data available on metabolomic changes in SGA children: one study identified changes in the metabolome in media conditioned by placental explants from pregnancies complicated by SGA (23). Seventy-nine metabolites were altered including aconitate, lithospermic acid, tryptophan, and oxoproline. Alterations of multiple metabolites, including amino acids, lipids, and myo-inositol, have been identified in the serum of piglets with

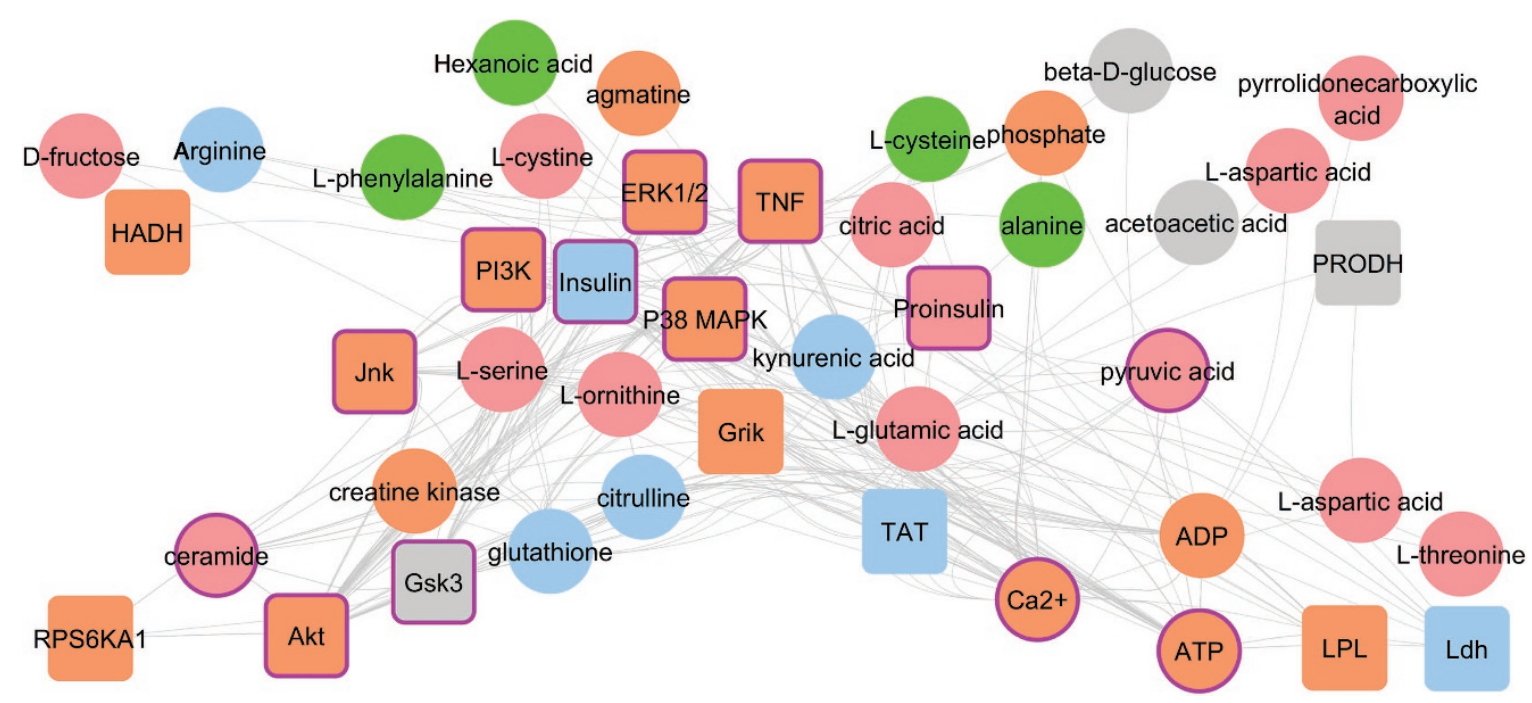

Figure 1. Interaction network of differences in metabolite regulation between control and small for gestational age patient cell lines. Nineteen metabolites were identified as differentially regulated between control and patient cells; these were used to define a network with inferred protein and metabolite interactions (Ingenuity Pathway Analysis Software (IPA)). Predicted activity in the network was calculated by derivation from the findings within the Ingenuity Knowledge Base database between the unknown molecule and its known neighbors (Molecule Activity Predictor in IPA). Purple highlight indicates involvement with insulin signaling (13/44). Red = predicted to be upregulated with darker red indicating greater upregulation. Green = predicted downregulated with darker green indicating greater downregulation. Orange = predicted activation with darker orange indicating greater activation. Blue $=$ predicted inhibition with darker blue indicating greater inhibition .

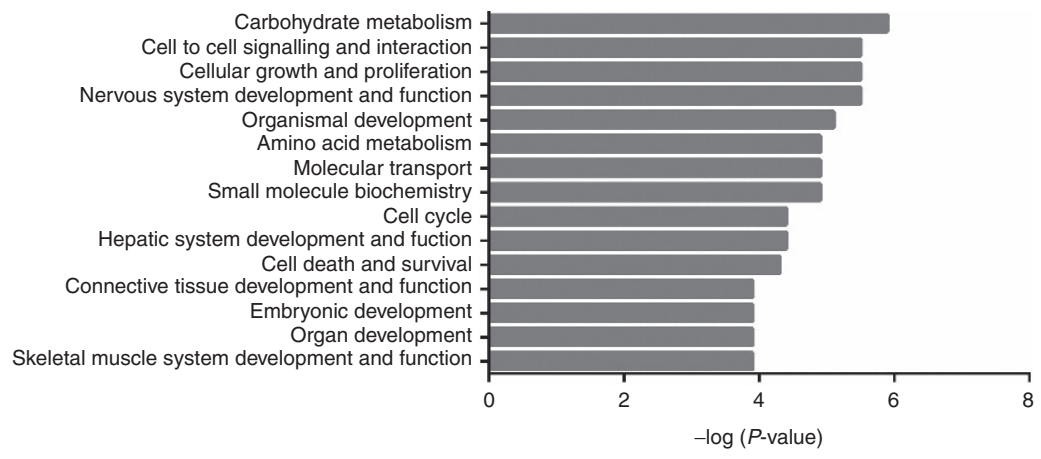

Figure 2. Biological functions associated with differences in metabolite regulation between control and small for gestational age patient cell lines. Biological functions ranked by $P$ value of Fisher's exact test (negative log). 


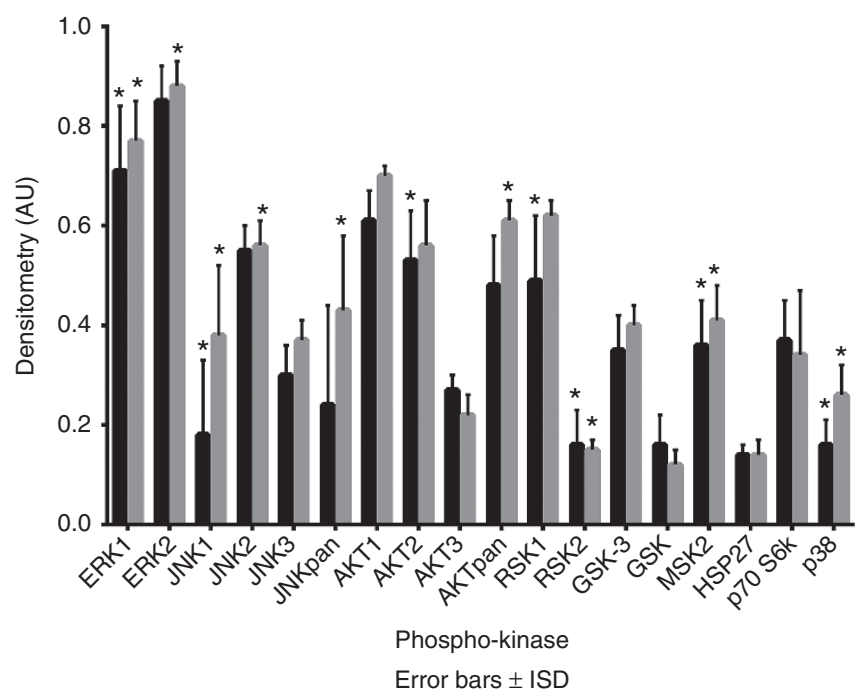

Figure 3. Phospho-kinase activation after stimulation with IGF-I in control and small for gestational age (SGA) patient cells. In control but not SGA cells, IGF-I preferentially activates ERK2 and JNK2 while in SGA but not control cells RSK2 is activated. ${ }^{*} P>0.05$ for difference between the IGF-I stimulated phospho-kinase activation (shown on graph) and baseline phospho-kinase activity (not shown). Black bars = SGA; gray bars = control.

intrauterine growth restriction (24). Dessì et al. (20) studied the urinary metabolome in intrauterine growth restricted (IUGR) and normal weight newborns and identified differences in the concentrations of myo-inositol and creatinine (both upregulated). An increase in myo-inositol was demonstrated in a second cohort of IUGR infants as well as in large for gestational age infants $(19,25)$. The IUGR infants from the studies of Dessì et al used a definition of weight at birth $<10$ th centile and so may have included in the IUGR group newborns who would be classed as normal based on a definition of SGA being a weight SDS $<-2$. Another study compared the metabolome from cord blood samples of SGA and AGA infants and identified increases in the amino acids proline, valine, isoleucine, glutamate, phenylalanine, and tryptophan (26). Work from our own laboratory studying the serum and urine metabolome of 33 SGA children (all age $>4$ y, 22 catch up and 11 noncatch-up) identified significant differences in myo-inositol (in urine), decanoic acid (in serum), glutamine (in serum), uric acid (in urine), and carnitine (in urine) (21). The most consistently identified metabolite altered in SGA/IUGR appears to be myo-inositol. With the exception of phenylalanine, the metabolites identified as being altered in SGA in this study are different to those identified in other studies, which represents the differences in examining biofluids (urine or serum) compared to the cellular metabolome.

A cell model was chosen for this study in order to allow examination of a snapshot of the intracellular metabolism as previous studies have focused on serum, urine, and conditioned media (all of which provide information on longer-term metabolic uptake and secretion). Fibroblasts were chosen as they have previously been shown to be a good model for studying growth disorders (15-17) and are easy to obtain via a skin biopsy. For studying the effects of being SGA on glucose, fat, or lipid metabolism, it may be better to dedifferentiate the fibroblasts into induced pluripotent stem cells and then redifferentiate the stem cells into adipocytes or hepatocytes. The growth and metabolic changes seen in short children born SGA represent long-term modifications to cellular processes. Studying a cultured fibroblast cell line rather than tissue avoided the possibility of local factors at the time of biopsy (e.g., trauma) influencing results.

In this study, there were decreases in 2-methyl-3-hydroxybutanoic acid and 3-methylpentanoic acid, which are both organic acids generated by isoleucine metabolism. Isoleucine is one of the three branched chain amino acids and increases in levels of the branched chain amino acids are linked to obesity and insulin resistance (27). A second observation was related to the depletion of the intracellular alanine pool either as a result of increased production of aspartic acid, glutamic acid, and ornithine or as a result of reduced conversion of pyruvate to alanine via alanine transferase. Alanine was present at a fourfold lower concentration in SGA subjects while aspartic acid was present at a fourfold higher concentration in the metabolic fingerprint and pyruvate at 1.4-fold higher concentration. Ornithine and glutamic acid, both products of alanine catabolism, were present at 1.4-fold higher concentrations in SGA subjects in the metabolic fingerprint. Thus, there is evidence for both reduced production and increased catabolism of alanine. Abnormalities in alanine and branched chain amino acids have been linked to cardiovascular disease as a rise in these metabolites is found in populations in China where there are increased rates of cardiovascular disease and obesity (28). Alterations in the levels of alanine, threonine, and the branched chain amino acids have also been found in infants born with weight of less than $1,500 \mathrm{~g}$ (this study included premature infants, hence not all infants will have been SGA) (29).

Biological network analysis of the footprint and fingerprint metabolomic data obtained in this study identified a network involving insulin and PI3K, ERK, and AKT. The biological functions significantly associated with the differentially regulated metabolites included cell growth, cell cycle, and carbohydrate metabolism. PI3K, ERK, and AKT are involved in the signal transduction pathways of both insulin and IGF-I receptors. Alterations in signal transduction of IGF-I and insulin are a plausible mechanism to cause both the growth and metabolic effects seen in SGA children. Increased activity within growthrelated pathways has previously been associated with short stature disorders such as Noonan syndrome (30), while inhibition of insulin signaling is seen in Donohue syndrome, also associated with growth restriction (31). This study therefore strengthens the suggestion that the cellular metabolic pathways involved in glucose regulation and growth are altered in SGA children. The phospho-kinase array identified differences between the control and SGA cell lines in the activation of growth-related signaling pathways and these were represented in the network analysis (ERK, JNK2, and RSK2).

This study has identified multiple metabolomic changes in fibroblasts from SGA children. Horgan et al. (32) identified a serum metabolomic profile in early pregnancy, which 


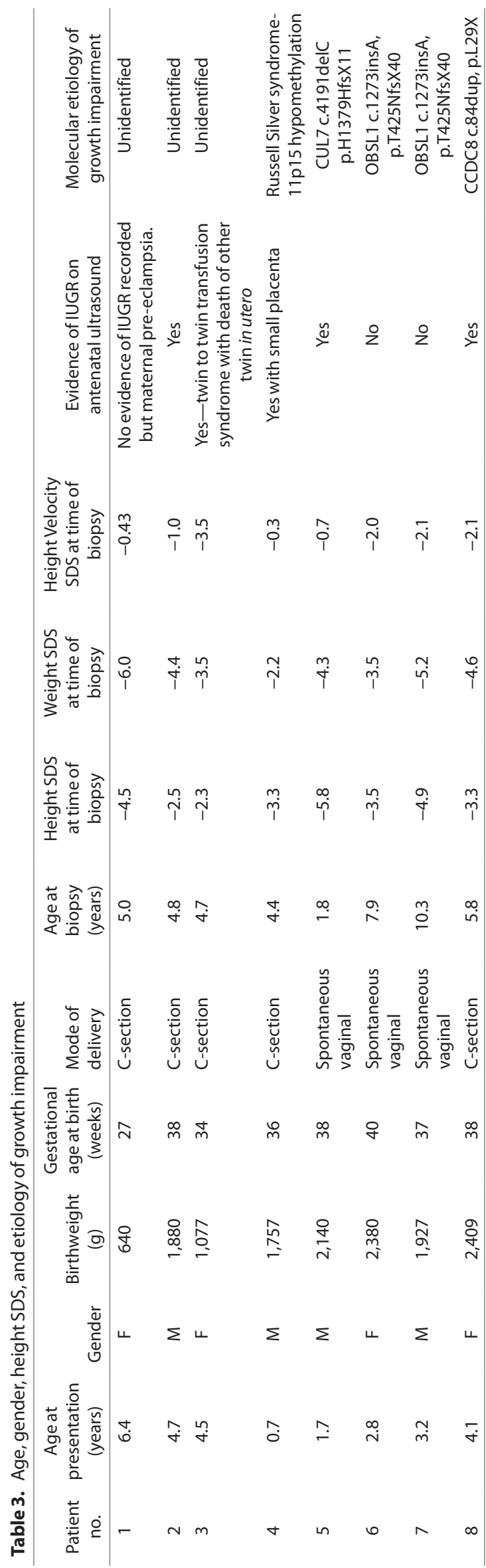

was predictive of the child being born SGA. Further work is required to determine if any of these metabolomic changes can be used either in utero or during the first few months of life to predict subsequent growth and metabolic health in SGA children. The limitations of the study include the small number of subjects and the use of GC-MS only. The application of liquid-chromatography-mass spectrometry would increase the coverage of metabolites detected. Evidence is presented for changes in growth and glucose metabolism pathways in skin fibroblast cell lines derived from SGA children. Further extensive studies are required to identify whether changes in the metabolome may correlate with growth phenotype, for instance, differentiating those SGA children who are destined either to experience inadequate catch-up growth, full catchup, or even excessive weight gain. In particular, future studies should focus on expanding this work into the clinical arena with prospective studies in babies born SGA.

In conclusion, we have identified intracellular metabolomic changes linked in network analysis to reduced insulin and IGF-I signal transduction in fibroblasts derived from short children born SGA.

\section{METHODS \\ Patients}

Patients were recruited from the regional Growth Clinic at the Royal Manchester Children's Hospital. They were eligible for inclusion where they were born SGA (birth weight SDS $\leq-2$ SD) and had either failure of catch-up growth with height SDS $\leq-2$ at $>2$ y of age or an identified genetic mutation associated with absence of catch-up growth. Seven patients were recruited on the basis of a height $<-2$ $\mathrm{SD}$ at $>2 \mathrm{y}$ of age with the remaining child recruited at $1.8 \mathrm{y}$ of age displaying no evidence of catch-up growth and having a CUL7 mutation, which is associated with absence of postnatal catch-up. All of our patients therefore had pathological growth impairment demonstrated by genetic mutations associated with growth failure $(n=5)$, evidence on intrauterine growth restriction on antenatal ultrasound $(n=5)$, and postnatal growth impairment $(n=8)$. Skin fibroblast cell lines were derived from three SGA patients with no defined etiology, one patient with Russell Silver syndrome (11p15 hypomethylation), four 3-M syndrome patients, and three control subjects. Including those with a defined as well as undefined etiology allowed us to assess whether their metabolome overall gives any indication of general growth and metabolic disorders in childhood. Biopsies were obtained from the forearm after application of EMLA cream (AstraZeneca, Macclesfield, UK). The 3-M patients included one male with a homozygous CUL7 mutation (c.4191delC p.H1379HfsX11), one male and one female (siblings) with a homozygous OBSL1 mutation (c.1273insA, p.T425NfsX40), and one female with a homozygous CCDC8 mutation (c.84dup, p.L29X). All children had exhibited significant failure of postnatal growth (see Table 3 ). The three control fibroblast cell lines (two male aged 4 and 9 y, one female aged 7 y) were derived from skin obtained during removal of skin tags from healthy normal statured children and was provided by the University of Manchester Centre for Genomic Medicine. All patients and control subjects were prepubertal at the time the skin samples were obtained.

Informed consent was obtained from parents, and the study was approved by the Central Manchester Research Ethics Committee (07/ Q1402/67 and 08/H1008/39) (North West Centre for Research Ethics Committees, Manchester, UK).

\section{Cell Culture}

Fibroblast cells were cultured in Dulbecco's modified Eagles medium (Invitrogen, Paisley, UK) supplemented to a final concentration with $10 \%$ fetal bovine serum (Invitrogen), 50 units $/ \mathrm{ml}$ penicillin, $50 \mu \mathrm{g} /$ $\mathrm{ml}$ streptomycin, $2 \mathrm{mmol} / \mathrm{l}$ glutamine and $2.5 \mu \mathrm{g} / \mathrm{ml}$ amphoterocin B 
(Invitrogen). Confluent cells passage eight were switched to serumfree media $24 \mathrm{~h}$ prior to sampling for metabolomics analysis.

\section{Metabolic Footprint Collection and Preparation}

Culture medium (defined as the metabolic footprint) was removed by aspiration and immediately stored at $-80^{\circ} \mathrm{C}$. Samples were prepared for analysis by lyophilizing $200 \mu \mathrm{l}$ aliquots (HETO VR MAXI vacuum centrifuge attached to a HETO CT/DW 60E cooling trap; Thermo Life Sciences, Basingstoke, UK).

\section{Metabolic Fingerprint Collection and Preparation}

Immediately following the removal of the metabolic footprint sample, the cells were rapidly washed twice with $5 \mathrm{ml}$ ice-cold phosphatebuffered saline to remove media contaminants. A quenching solution $\left(80 \%\right.$ methanol in water, $-40{ }^{\circ} \mathrm{C}$ ) was immediately added to the cells to suppress metabolism. The cells were released by scraping and the resulting suspension was subjected to three freeze/thaw cycles, using liquid nitrogen, to disrupt cell membranes and release the intracellular metabolome to the solution (defined as the metabolic fingerprint). The extraction solution was separated from the cellular biomass by centrifugation $(2,500 \mathrm{~g}$ for $5 \mathrm{~min})$. Aliquots $(1.5 \mathrm{ml})$ were lyophilized and then stored at $-80^{\circ} \mathrm{C}$ prior to further analysis.

\section{Gas Chromatography-Mass Spectrometry}

Lyophilized samples (a total of 66 footprint samples and 66 fingerprint samples) were chemically derivatised by addition of an O-methylhydroxylamine solution $\left(50 \mu \mathrm{l}, 20 \mathrm{mg} \cdot \mathrm{ml}^{-1}\right.$ in pyridine $60{ }^{\circ} \mathrm{C}$ for $30 \mathrm{~min}$ ) (Sigma-Aldrich, Gillingham, UK); followed by addition of $50 \mu \mathrm{l}$ MSTFA (N-acetyl-N-(trimethylsilyl)-trifluoroacetamide) (SigmaAldrich) and heating at $60^{\circ} \mathrm{C}$ for $30 \mathrm{~min} .20 \mu \mathrm{l}$ of a retention index solution (0.6 mg ml-1 $\mathrm{C}_{10}, \mathrm{C}_{12}, \mathrm{C}_{15}, \mathrm{C}_{19}$, and $\mathrm{C}_{22} \mathrm{n}$-alkanes) was added to the derivatised solution. Particulate matter was removed by centrifugation $(15 \mathrm{~min}, 13,3639 \mathrm{~g})$ followed by transfer of the supernatant to $300 \mu \mathrm{l}$ glass inserts placed in $2 \mathrm{ml}$ chromatography vials that were sealed with a polytetraflouroethylene/rubber septum containing screw cap.

Derivatized samples were analyzed on a 6890 gas chromatograph and 7890 autosampler (Agilent Technologies, Cheadle, UK) coupled to a Pegasus III electron impact mass spectrometer (Leco, Stockport, UK) as previously described (33).

Raw data files (.peg format) acquired from the GC-ToF-MS platform were directly processed by applying the ChromaTof v 2.25 software (Leco Corp, St Joseph, MI) as previously described (34). Data for each sample set (metabolic fingerprint and metabolic footprint) were integrated as a single dataset in.xls format for further data processing and analysis. Median values were calculated for data acquired for six technical replicates related to a single subject. All data were normalized to the total peak area ((peak area-metabolite/ total peak area-all metabolites $\left.)^{\star} 100\right)$. Metabolites were identified by comparison of retention index and electron impact-derived fragmentation mass spectrum to an in-house mass spectral library (35) or by comparison of the mass spectrum to the Golm Metabolome Database (36) or NIST08 mass spectral library (http://www.nist. gov/srd/nistla.cfm). Four different levels of reporting metabolite annotation or identification are available, as defined by The Metabolomics Standards Initiative (37). Level 1 identification was achieved if matching of RI and mass spectrum to a metabolite in the in-house library was achieved. Level 2 identification was achieved by matching to a metabolite present in Golm Metabolome Database or NIST08 libraries by mass spectrum only.

\section{Statistical Analysis}

Univariate statistical analysis was performed, using the nonparametric Mann-Whitney $U$-test to determine those metabolites showing a statistically significant difference $(P$ value $<0.05)$ between classes under observation.

\section{Analysis of Associated Biological Function}

Metabolic pathway enrichment analysis was performed using the MetaboAnalyst software $(22,38)$. All metabolites identified as statistically significant in metabolic footprint or metabolic fingerprint data were included. The Homo sapiens pathway was applied as well as the hypergeometric test (for over-representation analysis) and relativebetweeness centrality (for pathway topology analysis).
Network analysis of metabolomic data was performed to associate differences in metabolites between SGA and normal cells with all known metabolite and protein interactions, and hence, assess functional relevance. The Pubchem identifier of associated metabolites was mapped to its corresponding object in Ingenuity's Knowledge Base (Qiagen, Redwood City, CA). These molecules, called Network Eligible molecules, were overlaid onto a global molecular network developed from information contained in Ingenuity's Knowledge Base. Interaction Networks were then generated based on the function of these molecules using the ingenuity pathways analysis software algorithm. Network data was exported from ingenuity pathways analysis into Cytoscape 3.2.1 with edge bundling (39-42).

\section{Phospho-kinase Array}

Cells were serum starved for $24 \mathrm{~h}$ and then treated with or without IGF-I (100 ng/ml) (R\&D Systems, Abingdon, UK) for $15 \mathrm{~min}$. Lysates (a pool of four independent experiments) were applied to a human phospho Mitogen Activated Protein Kinase array (R\&D systems, Abingdon, UK) in accordance with the manufacturer's protocol. Densitometry was assessed with imageJ (NIH, Bethesda, MD) (43). Three control cell lines and three SGA cell lines were examined (not including RSS or 3-M syndrome).

\section{SUPPLEMENTARY MATERIAL}

Supplementary material is linked to the online version of the paper at http:// www.nature.com/pr

\section{STATEMENT OF FINANCIAL SUPPORT}

W.B.D. would like to thank BBSRC (Swindon, UK) for financial support of The Manchester Centre for Integrative Systems Biology (BBC0082191). I.B. was supported by an unrestricted educational grant from NovoNordisk (Bagsvaerd, Denmark). P.G.M. was supported by a Medical Research Council (Swindon, UK) Clinical Research Training Fellowship (G0700541).

Disclosure: P.G.M., W.B.D., M.B., A.W., A.S., G.C.B., and M.W. have nothing to declare. I.B. was the recipient of an unrestricted educational grant from NovoNordisk (Bagsvaerd, Denmark). P.E.C. has received lecture fees from NovoNordisk (Bagsvaerd, Denmark).

\section{REFERENCES}

1. Office for National Statistics. Births in England and Wales by Characteristics of Birth 2, 2013. http://www.ons.gov.uk/ons/dcp171778_384394.pdf.

2. Ananth CV, Vintzileos AM. Distinguishing pathological from constitutional small for gestational age births in population-based studies. Early Hum Dev 2009;85:653-8.

3. Wollmann HA. Intrauterine growth restriction: definition and etiology. Horm Res 1998;49 Suppl 2:1-6.

4. Clayton PE, Cianfarani S, Czernichow P, Johannsson G, Rapaport R, Rogol A. Management of the child born small for gestational age through to adulthood: a consensus statement of the International Societies of Pediatric Endocrinology and the Growth Hormone Research Society. J Clin Endocrinol Metab 2007;92:804-10.

5. Saenger P, Czernichow P, Hughes I, Reiter EO. Small for gestational age: short stature and beyond. Endocr Rev 2007;28:219-51.

6. Klingseisen A, Jackson AP. Mechanisms and pathways of growth failure in primordial dwarfism. Genes Dev 2011;25:2011-24.

7. Dunn WB, Broadhurst DI, Atherton HJ, Goodacre R, Griffin JL. Systems level studies of mammalian metabolomes: the roles of mass spectrometry and nuclear magnetic resonance spectroscopy. Chem Soc Rev 2011;40:387-426.

8. Dunn WB, Goodacre R, Neyses L, Mamas M. Integration of metabolomics in heart disease and diabetes research: current achievements and future outlook. Bioanalysis 2011;3:2205-22.

9. Issaq HJ, Fox SD, Chan KC, Veenstra TD. Global proteomics and metabolomics in cancer biomarker discovery. J Sep Sci 2011;34:3484-92.

10. Kenny LC, Broadhurst DI, Dunn W, et al.; Screening for Pregnancy Endpoints Consortium. Robust early pregnancy prediction of later preeclampsia using metabolomic biomarkers. Hypertension 2010;56:741-9.

11. Weiss RH, Kim K. Metabolomics in the study of kidney diseases. Nat Rev Nephrol 2012;8:22-33. 
12. Mikami T, Aoki M, Kimura T. The application of mass spectrometry to proteomics and metabolomics in biomarker discovery and drug development. Curr Mol Pharmacol 2012;5:301-16.

13. Dunn WB, Brown M, Worton SA, et al. Changes in the metabolic footprint of placental explant-conditioned culture medium identifies metabolic disturbances related to hypoxia and pre-eclampsia. Placenta 2009;30:974-80.

14. Boslem E, MacIntosh G, Preston AM, et al. A lipidomic screen of palmitate-treated MIN6 $\beta$-cells links sphingolipid metabolites with endoplasmic reticulum (ER) stress and impaired protein trafficking. Biochem J 2011;435:267-76.

15. Freeth JS, Ayling RM, Whatmore AJ, et al. Human skin fibroblasts as a model of growth hormone (GH) action in GH receptor-positive Laron's syndrome. Endocrinology 1997;138:55-61.

16. Freeth JS, Silva CM, Whatmore AJ, Clayton PE. Activation of the signal transducers and activators of transcription signaling pathway by growth hormone (GH) in skin fibroblasts from normal and GH binding proteinpositive Laron Syndrome children. Endocrinology 1998;139:20-8.

17. Silva CM, Kloth MT, Whatmore AJ, et al. GH and epidermal growth factor signaling in normal and Laron syndrome fibroblasts. Endocrinology 2002;143:2610-7.

18. Barker DJ. Adult consequences of fetal growth restriction. Clin Obstet Gynecol 2006;49:270-83.

19. Dessì A, Atzori L, Noto A, et al. Metabolomics in newborns with intrauterine growth retardation (IUGR): urine reveals markers of metabolic syndrome. J Matern Fetal Neonatal Med 2011;24 Suppl 2:35-9.

20. Dessì A, Marincola FC, Pattumelli MG, et al. Investigation of the ${ }^{1} \mathrm{H}-\mathrm{NMR}$ based urine metabolomic profiles of IUGR, LGA and AGA newborns on the first day of life. J Matern Fetal Neonatal Med 2014;27 Suppl 2:13-9.

21. Stevens A, Bonshek C, Whatmore A, et al. Insights into the pathophysiology of catch-up compared with non-catch-up growth in children born small for gestational age: an integrated analysis of metabolic and transcriptomic data. Pharmacogenomics J 2014;14:376-84.

22. Xia J, Mandal R, Sinelnikov IV, Broadhurst D, Wishart DS. MetaboAnalyst 2.0-a comprehensive server for metabolomic data analysis. Nucleic Acids Res 2012;40(Web Server issue):W127-33.

23. Horgan RP, Broadhurst DI, Dunn WB, et al. Changes in the metabolic footprint of placental explant-conditioned medium cultured in different oxygen tensions from placentas of small for gestational age and normal pregnancies. Placenta 2010;31:893-901.

24. He Q, Ren $\mathrm{P}$, Kong X, et al. Intrauterine growth restriction alters the metabonome of the serum and jejunum in piglets. Mol Biosyst 2011;7:2147-55.

25. Barberini L, Noto A, Fattuoni C, et al. Urinary metabolomics (GC-MS) reveals that low and high birth weight infants share elevated inositol concentrations at birth. J Matern Fetal Neonatal Med 2014;27 Suppl 2:20-6.

26. Favretto D, Cosmi E, Ragazzi E, et al. Cord blood metabolomic profiling in intrauterine growth restriction. Anal Bioanal Chem 2012;402:1109-21.
27. Newgard CB, An J, Bain JR, et al. A branched-chain amino acid-related metabolic signature that differentiates obese and lean humans and contributes to insulin resistance. Cell Metab 2009;9:311-26.

28. Yap IK, Brown IJ, Chan Q, et al. Metabolome-wide association study identifies multiple biomarkers that discriminate north and south Chinese populations at differing risks of cardiovascular disease: INTERMAP study. J Proteome Res 2010;9:6647-54.

29. Tea I, Le Gall G, Küster A, et al. 1H-NMR-based metabolic profiling of maternal and umbilical cord blood indicates altered materno-foetal nutrient exchange in preterm infants. PLoS One 2012;7:e29947.

30. Rauen KA. The RASopathies. Annu Rev Genomics Hum Genet 2013;14:355-69.

31. Falik Zaccai TC, Kalfon L, Klar A, et al. Two novel mutations identified in familial cases with Donohue syndrome. Mol Genet Genomic Med 2014;2:64-72.

32. Horgan RP, Broadhurst DI, Walsh SK, et al. Metabolic profiling uncovers a phenotypic signature of small for gestational age in early pregnancy. J Proteome Res 2011;10:3660-73.

33. O'Hagan S, Dunn WB, Brown M, Knowles JD, Kell DB. Closed-loop, multiobjective optimization of analytical instrumentation: gas chromatography/time-of-flight mass spectrometry of the metabolomes of human serum and of yeast fermentations. Anal Chem 2005;77:290-303.

34. Begley P, Francis-McIntyre S, Dunn WB, et al.; HUSERMET Consortium,. Development and performance of a gas chromatography-time-of-flight mass spectrometry analysis for large-scale nontargeted metabolomic studies of human serum. Anal Chem 2009;81:7038-46.

35. Brown M, Dunn WB, Dobson P, et al. Mass spectrometry tools and metabolite-specific databases for molecular identification in metabolomics. Analyst 2009;134:1322-32.

36. Kopka J, Schauer N, Krueger S, et al. GMD@CSB.DB: the Golm metabolome database. Bioinformatics 2005;21:1635-8.

37. Sumner LW, Amberg A, Barrett D, et al. Proposed minimum reporting standards for chemical analysis. Metabolomics 2007;3:211-21.

38. Xia J, Psychogios N, Young N, Wishart DS. MetaboAnalyst: a web server for metabolomic data analysis and interpretation. Nucleic Acids Res 2009;37(Web Server issue):W652-60.

39. Kohl M, Wiese S, Warscheid B. Cytoscape: software for visualization and analysis of biological networks. Methods Mol Biol 2011;696:291-303.

40. Shannon P, Markiel A, Ozier O, et al. Cytoscape: a software environment for integrated models of biomolecular interaction networks. Genome Res 2003;13:2498-504.

41. Smoot ME, Ono K, Ruscheinski J, Wang PL, Ideker T. Cytoscape 2.8: new features for data integration and network visualization. Bioinformatics 2011;27:431-2.

42. Su G, Morris JH, Demchak B, Bader GD. Biological network exploration with Cytoscape 3. Curr Protoc Bioinformatics 2014;47:8.13.1-24.

43. Schneider CA, Rasband WS, Eliceiri KW. NIH Image to ImageJ: 25 years of image analysis. Nat Methods 2012;9:671-5. 